\title{
Design Quadratic Patch and Cubic Patch of the Surface
}

\author{
R. B. M. Amer ${ }^{1}$, M. A. Abd El- Mageed ${ }^{2}$ \\ ${ }^{1,2}$ (Department of Mathematics and Phy. Engineering, Faculty of Engineering/Zagazig University, Egypt)
}

\begin{abstract}
This paper describes a new method for designing quadric patch and cubic patch of the surfaces and show how to use Mathematica programs for computations the equations of the patch of the surfaces. The surface with quadratic patches is defined through a given set of six points and the surface with cubic patches is defined through a given set of ten points.
\end{abstract}

Keywords: Quadric surfaces, Cubic surfaces, Coons patches, Bezier surface Patches, Mathematica program.

\section{Introduction}

Definition I.1: The 2-dimensoinal analog of a curve is a surface and the two dimensional generalization of a curve in $\mathfrak{R}^{\mathbf{n}}$ is a patch or local surface.

Definition I. 2: A patch or local surface is a differentiable mapping

$$
\mathbf{x}: U \rightarrow \mathfrak{R}^{\mathbf{n}},
$$

Where $\mathrm{u}$ is an open subset of $\mathfrak{R}^{2}$. More generally, if $\mathrm{A}$ is any subset of $\mathfrak{R}^{2}$, we say that a map $\mathbf{X}: \mathbf{A} \rightarrow \mathfrak{R}^{\mathbf{n}}$ is a patch provided that x can be extended differential mapping from u into $\mathfrak{R}^{\mathbf{n}}$, where $\mathrm{U}$ is an open set containing and we call $\mathrm{x}(\mathrm{U})$ or $\mathrm{x}(\mathrm{A})$ the trace of $\mathrm{x}$.

For example the patch sphere of radius a is defined on the closed rectangular $[0,2 \pi] \times\left[-\frac{\pi}{2}, \frac{\pi}{2}\right]$ but the differential extension to an open set containing $[0,2 \pi] \times\left[-\frac{\pi}{2}, \frac{\pi}{2}\right]$.

A patch can be written as an $\mathrm{n}$-tuple of functions

The partial derivatives $\mathrm{x}_{\mathrm{u}}$ of $\mathrm{x}$ with respect to $\mathrm{u}$ :

$$
\mathbf{x}(\mathbf{u}, \mathbf{v})=\left(\mathbf{x}_{1}(\mathbf{u}, \mathrm{v}), \ldots \ldots, \mathbf{x}_{\mathbf{n}}(\mathbf{u}, \mathrm{v})\right),
$$

$$
\mathbf{x}_{u}(\mathbf{u}, \mathbf{v})=\left(\frac{\partial \mathbf{x}_{1}}{\partial u}(\mathbf{u}, \mathbf{v}), \ldots \ldots . ., \frac{\partial \mathbf{x}_{n}}{\partial u}(\mathbf{u}, \mathbf{v})\right) .
$$

The other partial derivatives of $\mathrm{x}$ are $\mathbf{x}_{\mathbf{v}}, \mathbf{x}_{\mathrm{uu}}, \mathbf{x}_{\mathbf{v}}, \mathbf{x}_{\mathrm{vv}}$ see [3].

\section{Quadratic Surfaces}

The quadratic surfaces [1] are the second order algebraic equations and it is named surfaces $\mathrm{z}=\mathrm{f}(\mathrm{x}, \mathrm{y})$ which are denoted by the quadratic equations which has the form

$$
f(x, y, z)=a x^{2}+b y^{2}+c z^{2}+2 f x y+2 g x z+2 h y z+2 p x+2 q y+2 r z+d
$$

Where $a, b, c, d, e, f, g, h, p, q$ and $r$ are real constants and $x, y, z$ are variables and we can define a patch by $\mathbf{x}(\mathbf{u}, \mathbf{v})=(\mathbf{u}, \mathbf{v}, \mathbf{f}(\mathbf{u}, \mathbf{v}))$, where $u$ and $\mathrm{v}$ range over the domain of $\mathrm{f}$.

Examples of quadratic surfaces (see Fig.1) include the cone, cylinder, ellipsoid, elliptic cone, elliptic cylinder, elliptic hyperboloid, elliptic paraboloid, hyperbolic cylinder, hyperbolic paraboloid, paraboloid, sphere, and spheroid and these surfaces are said to be quadratic because all possible products of two of the variables $\mathrm{x}, \mathrm{y}, \mathrm{z}$ appear in quadratic equation. 


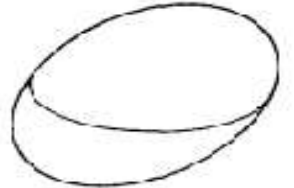

ellipsoid

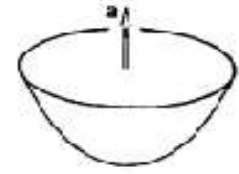

elliptic caraboloid

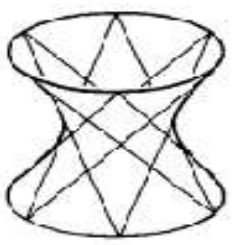

one-sheet hyperboloid

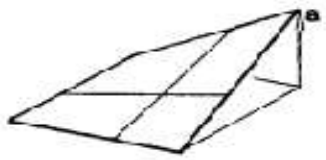

hyperbolic paraboloid

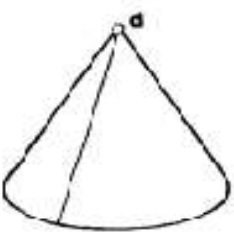

half-cone

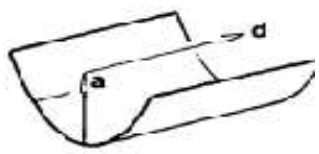

parapolic cylinder

ova! annullar

Fig 1. Some quadratic surfaces in $\mathrm{R}^{3}$

Since [5] a quadratic surface carries two families of straight lines called generators.

The types of surfaces determined from the both families of straight lines are:

1- The quadratic surfaces are doubly ruled or annular like hyperbolic paraboloid and hyperboloid of one sheet when the both families are real and different.

2- The quadratic surfaces are non ruled or oval like ellipsoid hyperboloid of two sheets and elliptic paraboloid when the both families are non real and different.

3- $\quad$ The quadratic surfaces are singly ruled and degenerate like cylindar and cone when the both families are coincide to one family.

Any plane section of quadratic is conic sections.

\section{Cubic Surfaces}

The Cubic surface are the third order algebraic equations in four homogenous variables $f(x, y, z, w)=$ 0. i.e., it is the vanishing set of homogenous polynomial of degree 3 in $\mathrm{P}^{\mathbf{3}}$ this mean it consist of all (x: $y: z: w)$ in $\mathrm{P}^{3}$ with

$$
\begin{aligned}
\mathbf{f}(x, y, z, w)= & a_{0} x^{3}+a_{1} y^{3}+a_{2} z^{3}+a_{3} x^{2} y+a_{4} x^{2} z+a_{5} x^{2} w+ \\
& +a_{6} y^{2} x+a_{7} y^{2} z+a_{8} y^{2} w+a_{9} z^{2} x+a_{10} z^{2} y+a_{11} z^{2} w+ \\
& +a_{12} w^{2} x+a_{13} w^{2} y+a_{14} w^{2} z+a_{15} x+a_{16} y+a_{17} z++a_{18} w+a_{19} .
\end{aligned}
$$

Examples of cubic surfaces (see Fig.2) include Clebsech diagonal surface which is designing by ten points and 27 lines and every three lines meet in a point [2] and also Cayley cubic surface which designing by four double points such that each one corresponds to a set of three points on a line in the plane.

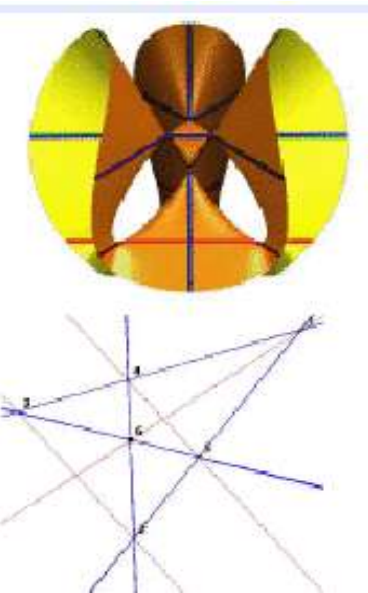

Clebsech diagonal surface

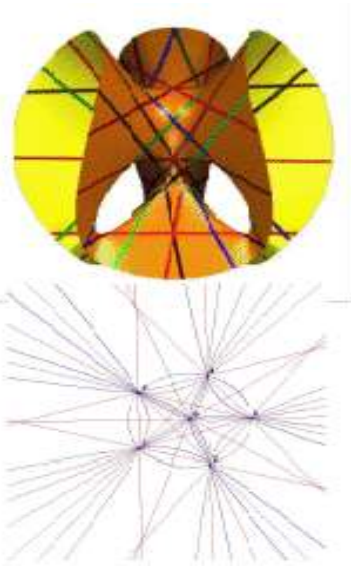

Cayley cubic surface

Fig.2 


\section{The Methods To Design Surface Patch}

There are many methods to design surfaces:

1- $\quad$ Ferguson(1963) [7] used parametric equations for the definitions of curves and surfaces in aircraft designed and Ferguson surface patch defined by this matrix.

$$
r=r(u, v)=\left[\begin{array}{llll}
1 & u & u^{2} & u^{3}
\end{array}\right]\left[\begin{array}{llll}
a_{00} & a_{01} & a_{02} & a_{03} \\
a_{10} & a_{11} & a_{12} & a_{13} \\
a_{20} & a_{21} & a_{22} & a_{23} \\
a_{30} & a_{31} & a_{32} & a_{33}
\end{array}\right]\left[\begin{array}{c}
1 \\
v \\
v^{2} \\
v^{3}
\end{array}\right] .
$$

2- $\quad$ Bezier(1970) has recombined the terms of Ferguson surface patch in away that make physical meaning of vector coefficients more apparent see [4], [6], [7] and Bezier surface patch defined by

$$
r=r(u, v)=\left[\begin{array}{llll}
1 & u & u^{2} & u^{3}
\end{array}\right] M B M^{T}\left[\begin{array}{c}
1 \\
v \\
v^{2} \\
v^{3}
\end{array}\right]
$$

Where

$$
M=\left[\begin{array}{cccc}
1 & 0 & 0 & 0 \\
-3 & 3 & 0 & 0 \\
3 & -6 & 3 & 0 \\
-1 & 3 & -3 & 1
\end{array}\right], \quad B=\left[\begin{array}{llll}
r_{00} & r_{01} & r_{02} & r_{03} \\
r_{10} & r_{11} & r_{12} & r_{13} \\
r_{20} & r_{21} & r_{22} & r_{23} \\
r_{30} & r_{31} & r_{32} & r_{33}
\end{array}\right]
$$

Bezier patch (see Fig. 3) is designed in terms of a characteristic polyhedron which is specified in terms of position vectors $r_{i j}$ of its 16 vertices.

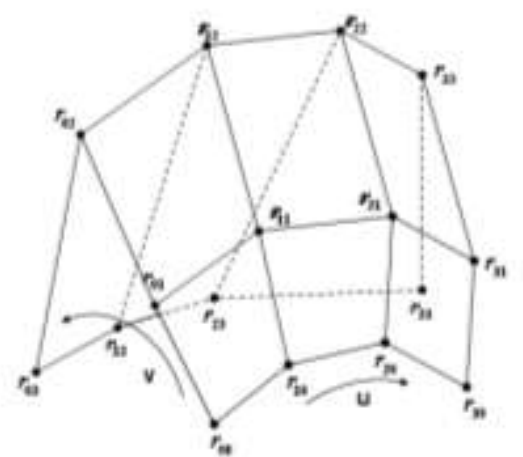

Fig. 3 Bezier surface patch

3- Coons (1967) [7] formed coons patches(see Fig. 4) which constructed from information given on its boundary and certain auxiliary scalar functions of $\mathrm{u}$ and $\mathrm{v}$ by dividing the surface into an assembly of topological rectangular patches each of which has its boundaries two u-curves and two v-curves.

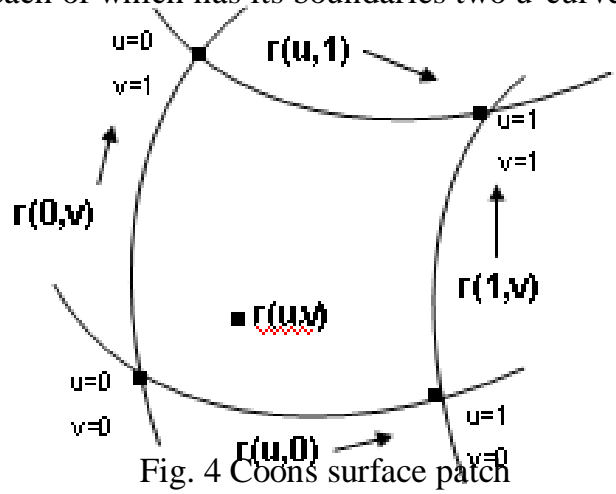


The Coons patch is defined by has four original curves as its boundaries and defined by this matrix

$$
\begin{aligned}
& r=r(u, v)=[(1-u) u]\left[\begin{array}{l}
r(\mathbf{0}, v) \\
r(1, v)
\end{array}\right]+\left[\begin{array}{ll}
r(u, 0) & r(u, 1)
\end{array}\right]\left[\begin{array}{c}
1-v \\
v
\end{array}\right]- \\
& -[(1-u) u]\left[\begin{array}{ll}
r(\mathbf{0 , 0}) & r(\mathbf{0 , 1}) \\
r(1,0) & r(1,1)
\end{array}\right]\left[\begin{array}{c}
1-v \\
v
\end{array}\right] \text {. }
\end{aligned}
$$

V. The New Method To Design Surface Patch

Let the graph of a real valued function of two variables $z=f(x, y)$ is a surface in $\mathfrak{R}^{\mathfrak{3}}$ (see Fig. 5). Assuming a surface patch is given as the graph of a function

Z

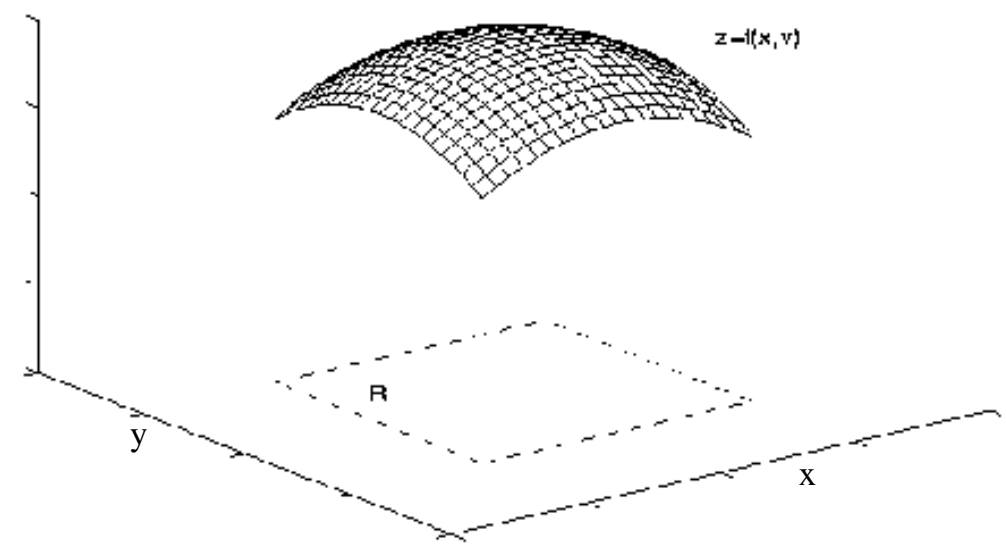

Fig. 5

For quadratic patch of the surface [8]:

\section{Quadratic Patch}

The general method is:

$$
z=z_{0}+\left(x-x_{0}\right) f_{x}+\left(y-y_{0}\right) f_{y}+\frac{\left(x-x_{0}\right)^{2}}{2} f_{x x}+\left(x-x_{0}\right)\left(y-y_{0}\right) f_{x y}+\frac{\left(y-y_{0}\right)^{2}}{2} f_{y y} .
$$

From this equation, there are five unknowns $f_{x}, f_{y}, f_{x y}, f_{x x}, f_{y y}$ with five equations. Form a set of five equations as follows:

$$
\begin{gathered}
\mathrm{z}_{1}-\mathrm{z}_{0}=\left(\mathrm{x}_{1}-\mathrm{x}_{0}\right) \mathrm{f}_{\mathrm{x}}+\left(\mathrm{y}_{1}-\mathrm{y}_{0}\right) \mathrm{f}_{\mathrm{y}}+\frac{\left(\mathrm{x}_{1}-\mathrm{x}_{0}\right)^{2}}{2} \mathrm{f}_{\mathrm{xx}}+\left(\mathrm{x}_{1}-\mathrm{x}_{0}\right)\left(\mathrm{y}_{1}-\mathrm{y}_{0}\right) \mathrm{f}_{\mathrm{xy}}+\frac{\left(\mathrm{y}_{1}-\mathrm{y}_{0}\right)^{2}}{2} \mathrm{f}_{\mathrm{yy}} \cdot \\
\mathrm{z}_{2}-\mathrm{z}_{0}=\left(\mathrm{x}_{2}-\mathrm{x}_{0}\right) \mathrm{f}_{\mathrm{x}}+\left(\mathrm{y}_{2}-\mathrm{y}_{0}\right) \mathrm{f}_{\mathrm{y}}+\frac{\left(\mathrm{x}_{2}-\mathrm{x}_{0}\right)^{2}}{2} \mathrm{f}_{\mathrm{xx}}+\left(\mathrm{x}_{2}-\mathrm{x}_{0}\right)\left(\mathrm{y}_{2}-\mathrm{y}_{0}\right) \mathrm{f}_{\mathrm{xy}}+\frac{\left(\mathrm{y}_{2}-\mathrm{y}_{0}\right)^{2}}{2} \mathrm{f}_{\mathrm{yy}} \cdot \\
\mathrm{z}_{3}-\mathrm{z}_{0}=\left(\mathrm{x}_{3}-\mathrm{x}_{0}\right) \mathrm{f}_{\mathrm{x}}+\left(\mathrm{y}_{3}-\mathrm{y}_{0}\right) \mathrm{f}_{\mathrm{y}}+\frac{\left(\mathrm{x}_{3}-\mathrm{x}_{0}\right)^{2}}{2} \mathrm{f}_{\mathrm{xx}}+\left(\mathrm{x}_{3}-\mathrm{x}_{0}\right)\left(\mathrm{y}_{3}-\mathrm{y}_{0}\right) \mathrm{f}_{\mathrm{xy}}+\frac{\left(\mathrm{y}_{3}-\mathrm{y}_{0}\right)^{2}}{2} \mathrm{f}_{\mathrm{yy}} \cdot \\
\mathrm{z}_{4}-\mathrm{z}_{0}=\left(\mathrm{x}_{4}-\mathrm{x}_{0}\right) \mathrm{f}_{\mathrm{x}}+\left(\mathrm{y}_{4}-\mathrm{y}_{0}\right) \mathrm{f}_{\mathrm{y}}+\frac{\left(\mathrm{x}_{4}-\mathrm{x}_{0}\right)^{2}}{2} \mathrm{f}_{\mathrm{xx}}+\left(\mathrm{x}_{4}-\mathrm{x}_{0}\right)\left(\mathrm{y}_{4}-\mathrm{y}_{0}\right) \mathrm{f}_{\mathrm{xy}}+\frac{\left(\mathrm{y}_{4}-\mathrm{y}_{0}\right)^{2}}{2} \mathrm{f}_{\mathrm{yy}} \cdot \\
\mathrm{z}_{5}-\mathrm{z}_{0}=\left(\mathrm{x}_{5}-\mathrm{x}_{0}\right) \mathrm{f}_{\mathrm{x}}+\left(\mathrm{y}_{5}-\mathrm{y}_{0}\right) \mathrm{f}_{\mathrm{y}}+\frac{\left(\mathrm{x}_{5}-\mathrm{x}_{0}\right)^{2}}{2} \mathrm{f}_{\mathrm{xx}}+\left(\mathrm{x}_{5}-\mathrm{x}_{0}\right)\left(\mathrm{y}_{5}-\mathrm{y}_{0}\right) \mathrm{f}_{\mathrm{xy}}+\frac{\left(\mathrm{y}_{5}-\mathrm{y}_{0}\right)^{2}}{2} \mathrm{f}_{\mathrm{yy}} .
\end{gathered}
$$

To solve these unknowns there exist six points as follows:

$\left(\mathrm{x}_{0}, \mathrm{y}_{0}, \mathrm{z}_{0}\right),\left(\mathrm{x}_{1}, \mathrm{y}_{1}, \mathrm{z}_{1}\right),\left(\mathrm{x}_{2}, \mathrm{y}_{2}, \mathrm{z}_{2}\right),\left(\mathrm{x}_{3}, \mathrm{y}_{3}, \mathrm{z}_{3}\right),\left(\mathrm{x}_{4}, \mathrm{y}_{4}, \mathrm{z}_{4}\right),\left(\mathrm{x}_{5}, \mathrm{y}_{5}, \mathrm{z}_{5}\right)$,

$\left(\mathrm{x}_{6}, \mathrm{y}_{6}, \mathrm{z}_{6}\right)$.

Take a point as an initial point $\left(\mathrm{x}_{0}, \mathrm{y}_{0}, \mathrm{z}_{0}\right)$.

We can be written the equations of unknowns by matrix form as follows: 


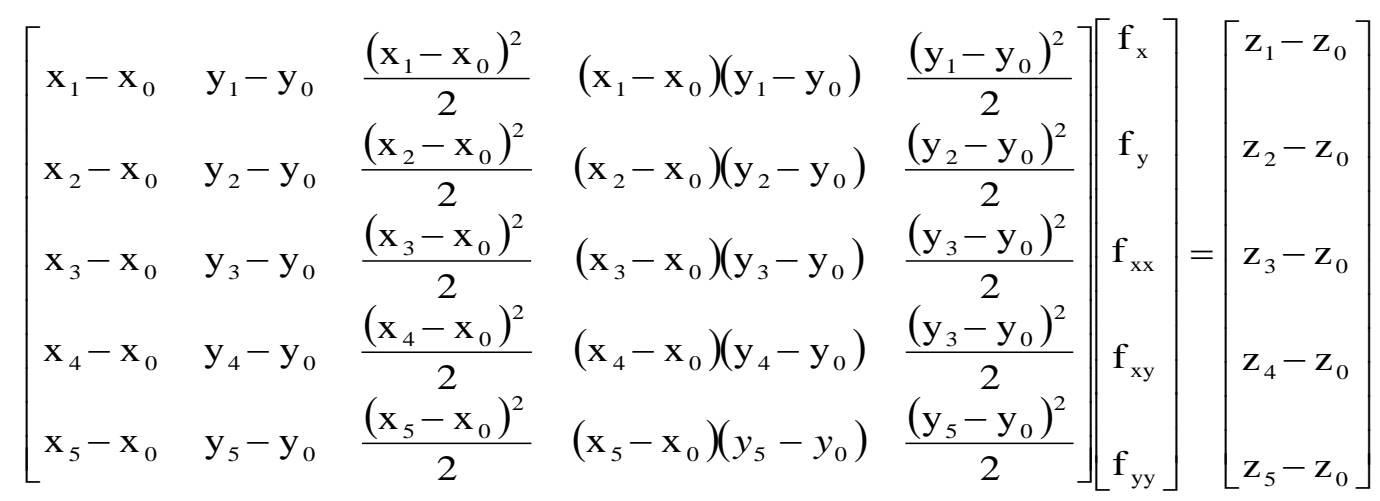

So we can solve this system to find the values of $f_{x}, f_{y}, f_{x x}, f_{x y}, f_{y y}$ and then substitute in above equation to find the equation of the patch of the surface.

By using Mathematica program to input above matrix

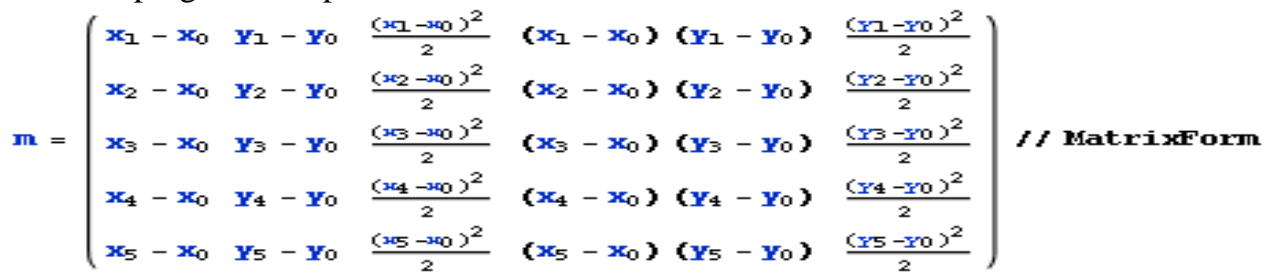

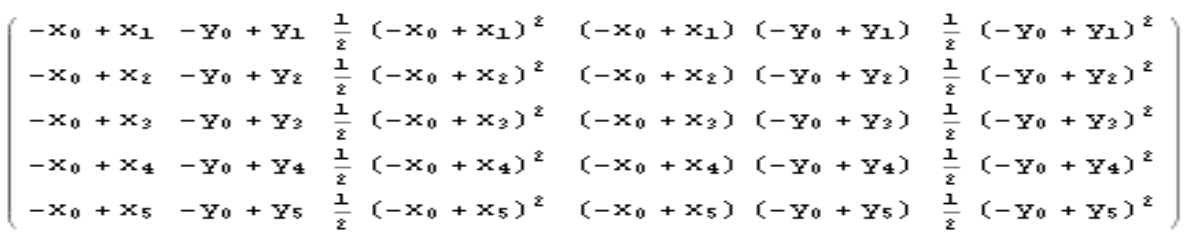

$$
\begin{aligned}
& \mathbf{v}=\left(\begin{array}{l}
z_{1}-z_{0} \\
z_{2}-z_{0} \\
z_{3}-z_{0} \\
z_{4}-z_{0} \\
z_{5}-z_{0}
\end{array}\right) \\
& \left\{\left\{-z_{0}+z_{1}\right\},\left\{-z_{0}+z_{2}\right\},\left\{-z_{0}+z_{3}\right\},\left\{-z_{0}+z_{4}\right\},\left\{-z_{0}+z_{5}\right\}\right\} \\
& \mathbf{s}=\left(\begin{array}{c}
\mathbf{f}_{\mathrm{xx}} \\
\mathbf{f}_{Y} \\
\mathbf{f}_{\mathrm{xY}} \\
\mathbf{f}_{\mathrm{xax}} \\
\mathbf{f}_{Y Y}
\end{array}\right) \\
& \left\{\left\{f_{x}\right\},\left\{f_{y}\right\},\left\{f_{x y}\right\},\left\{f_{x x}\right\},\left\{f_{y y}\right\}\right\}
\end{aligned}
$$

Let it is required to design a quadratic patch of a surface through the points:

$(0,0,3),(4,1,5),(-2,2,2),(1,4,6),(3,6,3),(6,3,1)$.

Using Mathematica program to inter these points:

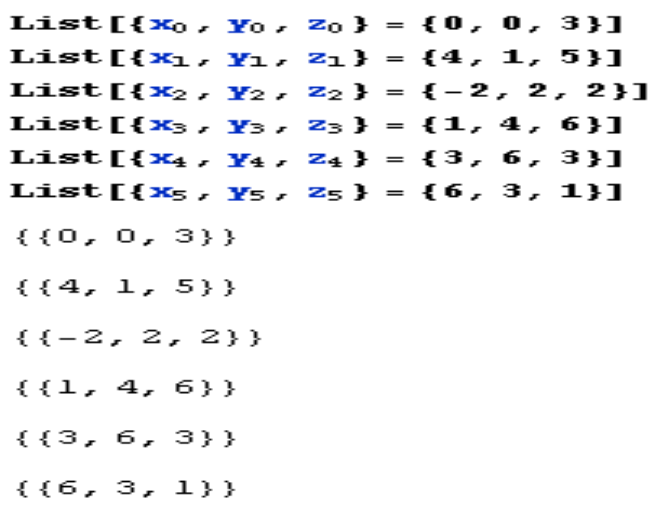

There are two methods to solve the linear system $\mathrm{m} . \mathrm{v}=\mathrm{s}$ by using Mathematica program. 
The first method:

Since we interred the six points so from mathematica program we can get without any calculations and also matrix $\mathrm{v}$ as follows:

$$
\begin{aligned}
& M=\left(\begin{array}{llllll}
x_{1}-x_{0} & Y_{1}-Y_{0} & \frac{\left(x_{1}-x_{0}\right)^{2}}{2} & \left(X_{1}-x_{0}\right)\left(Y_{1}-Y_{0}\right) & \frac{\left(Y_{1}-Y_{0}\right)^{2}}{2} \\
x_{2}-x_{0} & Y_{2}-Y_{0} & \frac{\left(x_{2}-x_{0}\right)^{2}}{2} & \left(X_{2}-x_{0}\right)\left(Y_{2}-Y_{0}\right) & \frac{\left(Y_{2}-Y_{0}\right)^{2}}{2} \\
x_{5}-x_{0} & Y_{3}-Y_{0} & \frac{\left(x_{5}-x_{0}\right)^{2}}{2} & \left(X_{3}-x_{0}\right)\left(Y_{3}-Y_{0}\right) & \frac{\left(Y_{3}-Y_{0}\right)^{2}}{2} \\
x_{4}-x_{0} & Y_{4}-Y_{0} & \frac{\left(x_{4}-x_{0}\right)^{2}}{2} & \left(X_{4}-x_{0}\right)\left(Y_{4}-Y_{0}\right) & \frac{\left(Y_{4}-Y_{0}\right)^{2}}{2} \\
x_{5}-x_{0} & Y_{5}-Y_{0} & \frac{\left(x_{5}-x_{0}\right)^{2}}{2} & \left(X_{5}-x_{0}\right)\left(Y_{5}-Y_{0}\right) & \frac{\left(Y_{5}-Y_{0}\right)^{2}}{2}
\end{array}\right) \text { MatrixForm } \\
& \left(\begin{array}{ccccc}
4 & 1 & 8 & 4 & \frac{1}{z} \\
-2 & 2 & 2 & -4 & \frac{2}{2} \\
1 & 4 & \frac{1}{z} & 4 & 8 \\
3 & 6 & \frac{9}{2} & 18 & 18 \\
6 & 3 & 18 & 18 & \frac{9}{z}
\end{array}\right) \\
& y=\left(\begin{array}{l}
z_{1}-z_{0} \\
z_{2}-z_{0} \\
z_{3}-z_{0} \\
z_{4}-z_{0} \\
z_{5}-z_{0}
\end{array}\right) \\
& \{\{2\},\{-1\},\{3\},\{0\},\{-2\}\}
\end{aligned}
$$

And then using linear solve command to get the unknowns

$\mathrm{s}=$ LinearSol ve $[\mathrm{m}, \mathrm{v}]$
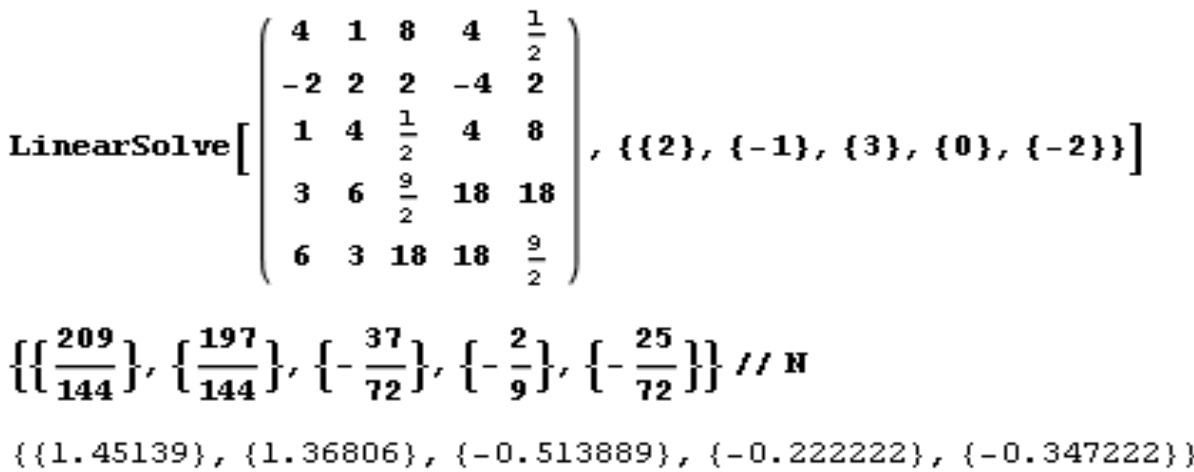

Then $\mathrm{f}_{\mathrm{x}}=1.45139, \mathrm{f}_{\mathrm{y}}=1.36806, \mathrm{f}_{\mathrm{xx}}=-0.513889, \mathrm{f}_{\mathrm{xy}}=-0.222222, \mathrm{f}_{\mathrm{yy}}=-0.347222$

From mathematica program substitute these values in the equation of quadratic surface patch we get:

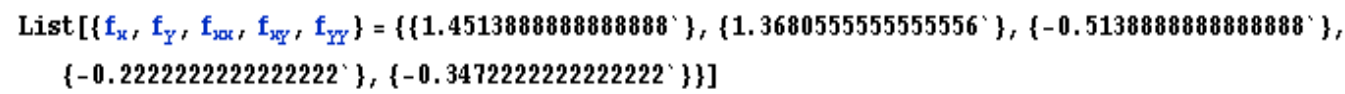

Then the equation of the quadratic patch of the surface from mathematica program is:

$$
\begin{gathered}
\mathrm{z}=3+1.4513888888888888^{\prime} \mathrm{x}-0.2569444444444444^{\prime} \mathrm{x}^{2}+1.36805555555555566^{\prime} \mathrm{y}- \\
0.2222222222222222^{\prime} \mathrm{x} \mathrm{y}-0.1736111111111111^{\prime} \mathrm{y}^{2}
\end{gathered}
$$


Or numerically:

$$
\mathrm{z}=3+1.45139 \mathrm{x}+1.36806 \mathrm{y}-0.2569445 \mathrm{x}^{2}-0.2222222222 \mathrm{xy}-\quad 0.173611 \mathrm{y}^{2} .
$$

\section{The Second method:}

We use RowReduce command to get the five unknowns
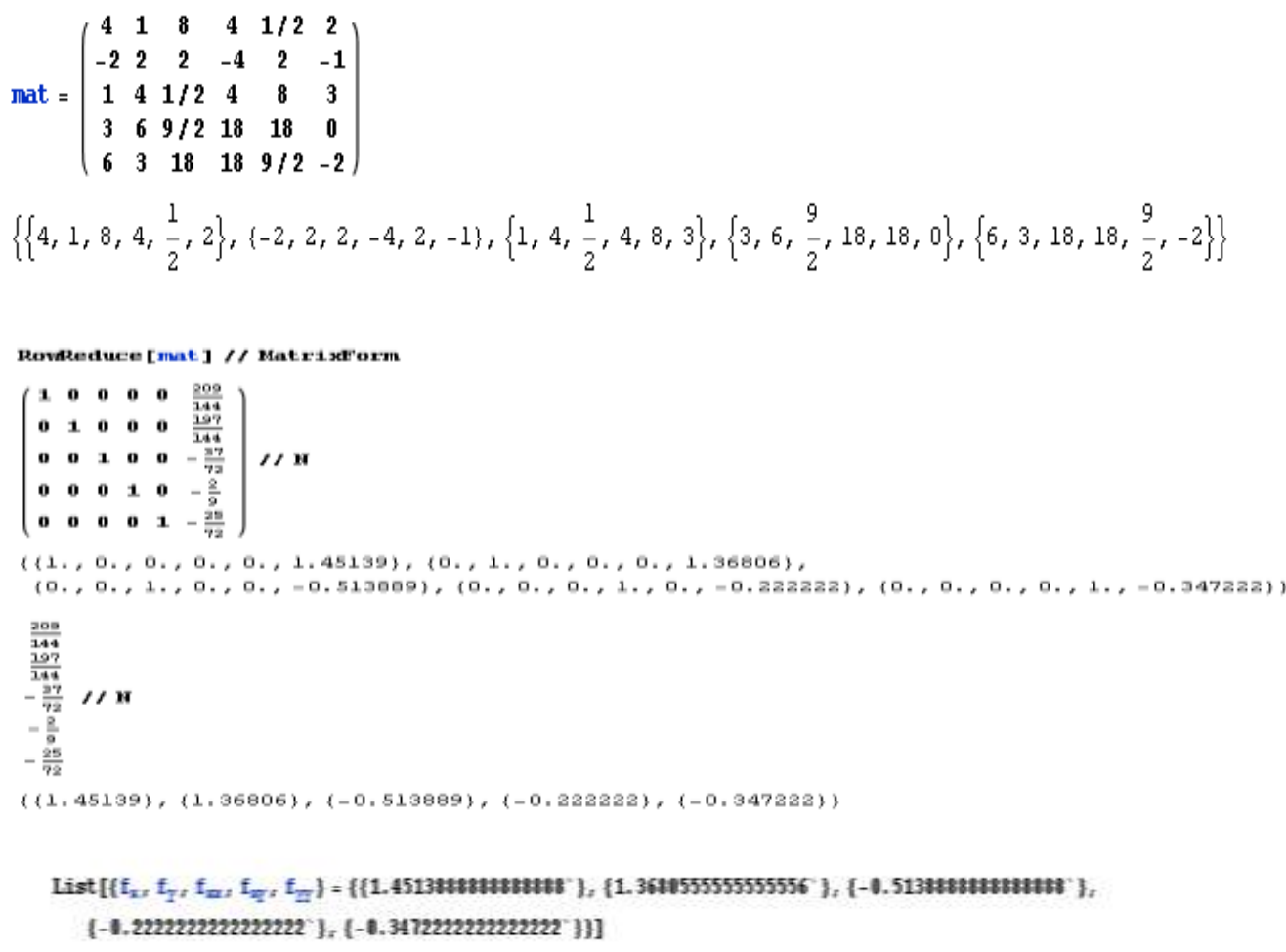

We get the same results

$$
\mathrm{z}=3+1.45139 \mathrm{x}+1.36806 \mathrm{y}-0.2569445 \mathrm{x}^{2}-0.2222222222 \mathrm{xy}-\quad 0.173611 \mathrm{y}^{2} .
$$

The program listed above help us to compute quadratic patch of the surface for any six points.

For cubic patch of the surface:

\section{Cubic Patch}

The general method:

$$
\begin{aligned}
z= & z_{0}+\left(x-x_{0}\right) f_{x}+\left(y-y_{0}\right) f_{y}+\frac{\left(x-x_{0}\right)^{2}}{2} f_{x x}+\left(x-x_{0}\right)\left(y-y_{0}\right) f_{x y}+\frac{\left(y-y_{0}\right)^{2}}{2} f_{y y}+ \\
& +\frac{\left(x-x_{0}\right)^{3}}{6} f_{x x x}+\frac{\left(x-x_{0}\right)^{2}\left(y-y_{0}\right)}{2} f_{x x y}+\frac{\left(y-y_{0}\right)^{2}\left(x-x_{0}\right)}{2} f_{y y x}+\frac{\left(y-y_{0}\right)^{3}}{6} f_{y y y} \cdot
\end{aligned}
$$

From this equation, there are nine unknowns $f_{x}, f_{y}, f_{x y}, f_{x x}, f_{y y}, f_{x x x}, f_{x x y}, f_{x y y}, f_{y y y}$ with nine equations. To solve these unknowns there exist ten points as follows: 
$\left(\mathrm{x}_{0}, \mathrm{y}_{0}, \mathrm{z}_{0}\right),\left(\mathrm{x}_{1}, \mathrm{y}_{1}, \mathrm{z}_{1}\right),\left(\mathrm{x}_{2}, \mathrm{y}_{2}, \mathrm{z}_{2}\right),\left(\mathrm{x}_{3}, \mathrm{y}_{3}, \mathrm{z}_{3}\right),\left(\mathrm{x}_{4}, \mathrm{y}_{4}, \mathrm{z}_{4}\right),\left(\mathrm{x}_{5}, \mathrm{y}_{5}, \mathrm{z}_{5}\right)$,

$\left(\mathrm{x}_{6}, \mathrm{y}_{6}, \mathrm{z}_{6}\right),\left(\mathrm{x}_{7}, \mathrm{y}_{7}, \mathrm{z}_{7}\right),\left(\mathrm{x}_{8}, \mathrm{y}_{8}, \mathrm{z}_{8}\right),\left(\mathrm{x}_{9}, \mathrm{y}_{9}, \mathrm{z}_{9}\right)$.

Take a point as an initial point $\left(\mathrm{x}_{0}, \mathrm{y}_{0}, \mathrm{z}_{0}\right)$.

We can be written the equations of unknowns by matrix form as follows:

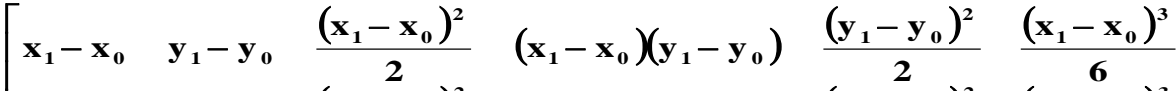

$$
\begin{aligned}
& x_{2}-x_{0} \quad y_{2}-y_{0} \frac{\left(x_{2}-x_{0}\right)^{2}}{2} \quad\left(x_{2}-x_{0}\right)\left(y_{2}-y_{0}\right) \frac{\left(y_{2}-y_{0}\right)^{2}}{2} \frac{\left(x_{2}-x_{0}\right)^{3}}{6} \\
& x_{3}-x_{0} \quad y_{3}-y_{0} \frac{\left(x_{3}-x_{0}\right)^{2}}{2}\left(x_{3}-x_{0}\right)\left(y_{3}-y_{0}\right) \frac{\left(y_{3}-y_{0}\right)^{2}}{2} \frac{\left(x_{3}-x_{0}\right)^{3}}{6}
\end{aligned}
$$

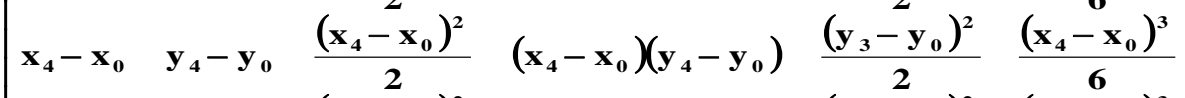

$$
\begin{aligned}
& x_{5}-x_{0} \quad y_{5}-y_{0} \frac{\left(x_{5}-x_{0}\right)^{2}}{2}\left(x_{5}-x_{0}\right)\left(y_{5}-y_{0}\right) \frac{\left(y_{5}-y_{0}\right)^{2}}{2} \frac{\left(x_{5}-x_{0}\right)^{3}}{6} \\
& x_{6}-x_{0} \quad y_{6}-y_{0} \frac{\left(x_{6}-x_{0}\right)^{2}}{2}\left(x_{6}-x_{0}\right)\left(y_{6}-y_{0}\right) \frac{\left(y_{6}-y_{0}\right)^{2}}{2} \quad \frac{\left(x_{6}-x_{0}\right)^{3}}{6} \\
& x_{7}-x_{0} \quad y_{7}-y_{0} \frac{\left(x_{7}-x_{0}\right)^{2}}{2}\left(x_{7}-x_{0}\right)\left(y_{7}-y_{0}\right) \frac{\left(y_{7}-y_{0}\right)^{2}}{2} \frac{\left(x_{7}-x_{0}\right)^{3}}{6} \\
& x_{8}-x_{0} \quad y_{8}-y_{0} \frac{\left(x_{8}-x_{0}\right)^{2}}{2}\left(x_{8}-x_{0}\right)\left(y_{8}-y_{0}\right) \frac{\left(y_{8}-y_{0}\right)^{2}}{2} \frac{\left(x_{8}-x_{0}\right)^{3}}{6}
\end{aligned}
$$

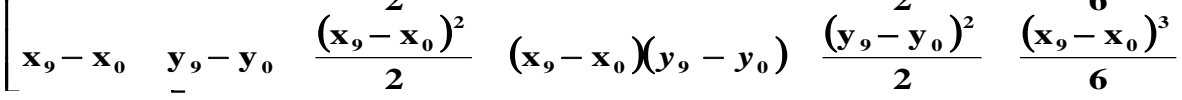

$$
\begin{aligned}
& \left.\frac{\left(\mathbf{x}_{1}-\mathbf{x}_{0}\right)^{2}\left(\mathbf{y}_{1}-\mathbf{y}_{0}\right)}{2} \quad \frac{\left(y_{1}-y_{0}\right)^{2}\left(x_{1}-x_{0}\right)}{2} \quad \frac{\left(y_{1}-y_{0}\right)^{3}}{6}\right] \\
& \begin{array}{llll}
\frac{\left(x_{2}-x_{0}\right)^{2}\left(y_{2}-y_{0}\right)}{2} & \frac{\left(y_{2}-y_{0}\right)^{2}\left(x_{2}-x_{0}\right)}{2} & \frac{\left(y_{2}-y_{0}\right)^{3}}{6} \\
\frac{\left(x_{3}-x_{0}\right)^{2}\left(y_{3}-y_{0}\right)}{2} & \frac{\left(y_{3}-y_{0}\right)^{2}\left(x_{3}-x_{0}\right)}{2} & \frac{\left(y_{3}-y_{0}\right)^{3}}{6}
\end{array} \\
& \frac{\left(\mathbf{x}_{4}-\mathbf{x}_{0}\right)^{2}\left(\mathbf{y}_{4}-y_{0}\right)}{2} \quad \frac{\left(y_{4}-y_{0}\right)^{2}\left(x_{4}-x_{0}\right)}{2} \quad \frac{\left(y_{4}-y_{0}\right)^{3}}{6} \\
& \frac{\left(\mathbf{x}_{5}-\mathbf{x}_{0}\right)^{2}\left(\mathbf{y}_{5}-\mathbf{y}_{0}\right)}{2} \quad \frac{\left(y_{5}-y_{0}\right)^{2}\left(x_{5}-x_{0}\right)}{2} \quad \frac{\left(y_{5}-y_{0}\right)}{6} \\
& \left.\begin{array}{llll}
\frac{\left(x_{6}-x_{0}\right)^{2}\left(y_{6}-y_{0}\right)}{2} & \frac{\left(y_{6}-y_{0}\right)^{2}\left(x_{6}-x_{0}\right)}{2} & \frac{\left(y_{6}-y_{0}\right)^{3}}{6} \\
\frac{\left(x_{7}-x_{0}\right)^{2}\left(y_{7}-y_{0}\right)}{2} & \frac{\left(y_{7}-y_{0}\right)^{2}\left(x_{7}-x_{0}\right)}{2} & \frac{\left(y_{7}-y_{0}\right)^{3}}{6} \\
\frac{\left(x_{8}-x_{0}\right)^{2}\left(y_{8}-y_{0}\right)}{2} & \frac{\left(y_{8}-y_{0}\right)^{2}\left(x_{8}-x_{0}\right)}{2} & \frac{\left(y_{8}-y_{0}\right)^{3}}{6}
\end{array}\right]\left[\begin{array}{l}
f_{x x x} \\
f_{x x x y} \\
f_{x y y} \\
f_{y y y}
\end{array}\right]\left[\begin{array}{l}
z_{6}-z_{0} \\
z_{7}-z_{0} \\
z_{8}-z_{0} \\
z_{9}-z_{0}
\end{array}\right] \\
& \frac{\left(x_{9}-x_{0}\right)^{2}\left(y_{9}-y_{0}\right)}{2} \quad \frac{\left(y_{9}-y_{0}\right)^{2}\left(x_{9}-x_{0}\right)}{2} \quad \frac{\left(y_{9}-y_{0}\right)^{3}}{6}
\end{aligned}
$$

So we can solve this system to find the values of nine unknowns $f_{x}, f_{y}, f_{x y}, f_{x x}, f_{y y}, f_{x x x}, f_{x x y}, f_{x y y}, f_{y y y}$ and then substitute in above equation to find the equation of the patch of the surface. By using Mathematica program to input above matrix 

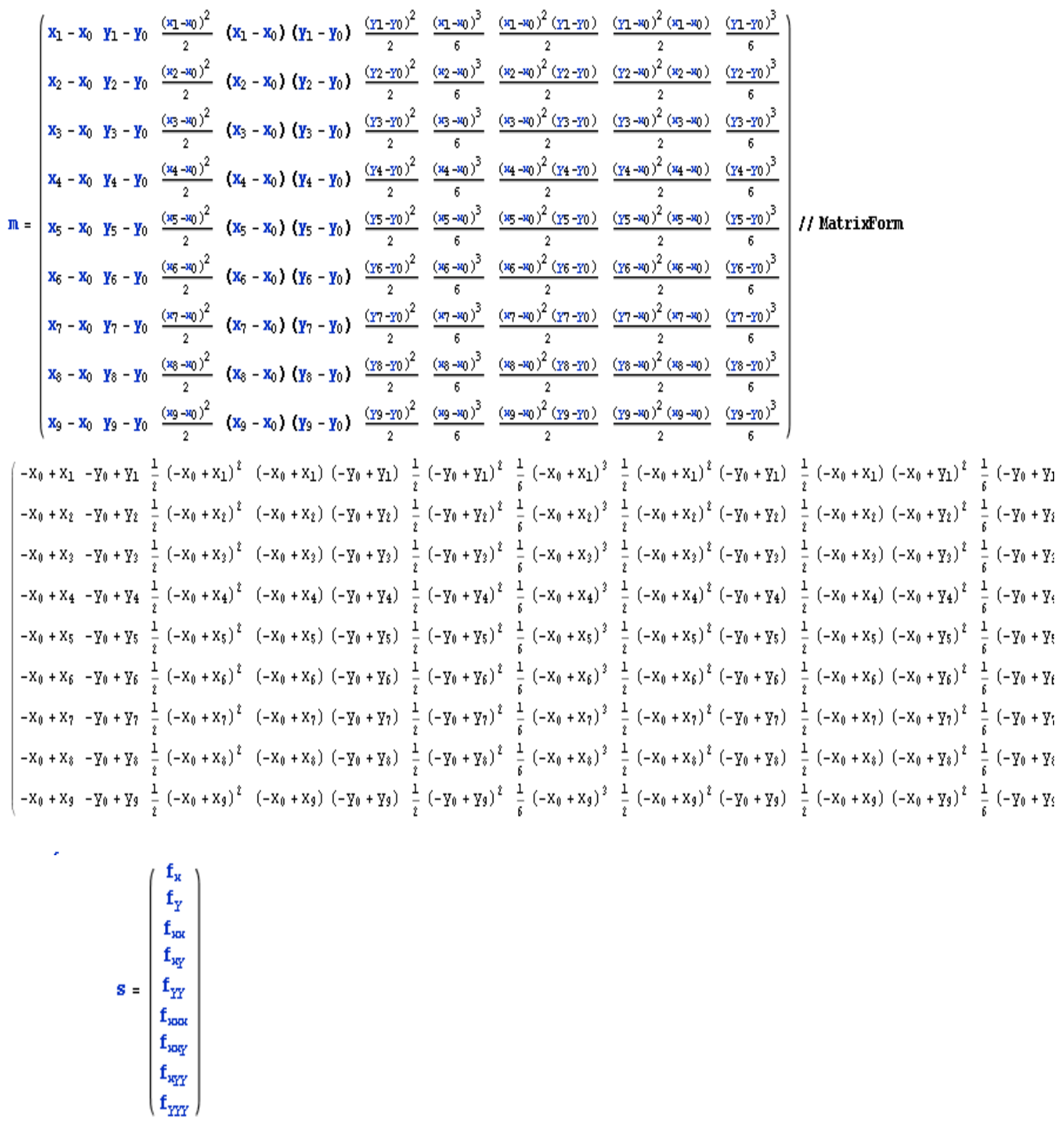

$\left\{\left\{\mathrm{f}_{\mathrm{x}}\right\},\left\{\mathrm{f}_{\mathrm{y}}\right\},\left\{\mathrm{f}_{\mathrm{xx}}\right\},\left\{\mathrm{f}_{\mathrm{xxy}}\right\},\left\{\mathrm{f}_{\mathrm{yy}}\right\},\left\{\mathrm{f}_{\mathrm{xxx}}\right\},\left\{\mathrm{f}_{\mathrm{xxy}}\right\},\left\{\mathrm{f}_{\mathrm{x} y \mathrm{y}}\right\},\left\{\mathrm{f}_{\mathrm{wyy}}\right\}\right\}$

$\mathrm{V}=\left(\begin{array}{l}z_{1}-z_{0} \\ z_{2}-z_{0} \\ z_{3}-z_{0} \\ z_{4}-z_{0} \\ z_{5}-z_{0} \\ z_{6}-z_{0} \\ z_{7}-z_{0} \\ z_{8}-z_{0} \\ z_{9}-z_{0}\end{array}\right)$

$\left\{\left\{-z_{0}+z_{1}\right\},\left\{-z_{0}+z_{2}\right\},\left\{-z_{0}+z_{3}\right\},\left\{-z_{0}+z_{4}\right\},\left\{-z_{0}+z_{5}\right\},\left\{-z_{0}+z_{6}\right\},\left\{-z_{0}+z_{7}\right\},\left\{-z_{0}+z_{0}\right\},\left\{-z_{0}+z_{9}\right\}\right\}$

Let it is required to design a cubic patch of a surface through the ten points:

$(0,0,3),(4,1,5),(-2,2,2),(1,4,6),(3,6,3),(6,3,1),(-1,5,7)$,

$(-3,-3,3),(2,-1,5),(5,-2,8)$.

Using mathematica program to inter ten points as follows 


$$
\begin{aligned}
& \operatorname{Limt}\left[\left(x_{0}, y_{0}, \square_{0}\right)=(0,0,3)\right] \\
& \text { Lise }\left[\left(x_{2}, y_{2}, x_{2}\right)=(-2,2,2)\right] \\
& \text { Liet }\left[\left(x_{4}, y_{4}, x_{4}\right)=(3,6,3)\right] \\
& \text { List }\left[\left(x_{6}, y_{6}, x_{6}\right)=(-1,3,7)\right] \\
& \text { Liat }\left[\left(x_{\theta}, y_{\theta}, x_{\theta}\right)=(2,-1,5)\right] \\
& ((0,0,3)) \\
& ((4,1,5)) \\
& ((-2,2,2)) \\
& (1,4,6)) \\
& ((3,6,3)) \\
& ((6,3,1)) \\
& ((-1,3,7)) \\
& ((-3,-3,3)) \\
& ((2,-1,5)) \\
& ((5,-2,6))
\end{aligned}
$$$$
\text { List }\left[\left(x_{1}, y_{1}, x_{1}\right)=(4,1,5)\right]
$$$$
\text { Liat }\left[\left(x_{3}, y_{3}, x_{3}\right)=(1,4,6)\right]
$$$$
\text { List }\left[\left(x_{5}, y_{5}, y_{5}\right)=(6,3,1)\right]
$$$$
\text { Liet }\left[\left(x_{7}, y_{7}, x_{7}\right)=(-3,-3,3)\right]
$$$$
\text { Liet }\left[\left(x_{9}, y_{9}, x_{9}\right)=(5,-2,8)\right]
$$

Then solve the linear system $m . s=v$ by using Mathematica program to get the nine unknowns $f_{x}, f_{y}, f_{x y}, f_{x x}$, $f_{y y}, f_{x x x}, f_{x x y}, f_{x y y}, f_{y y y}$ and then substitute the results in the equation of cubic patch of the surface.

Then
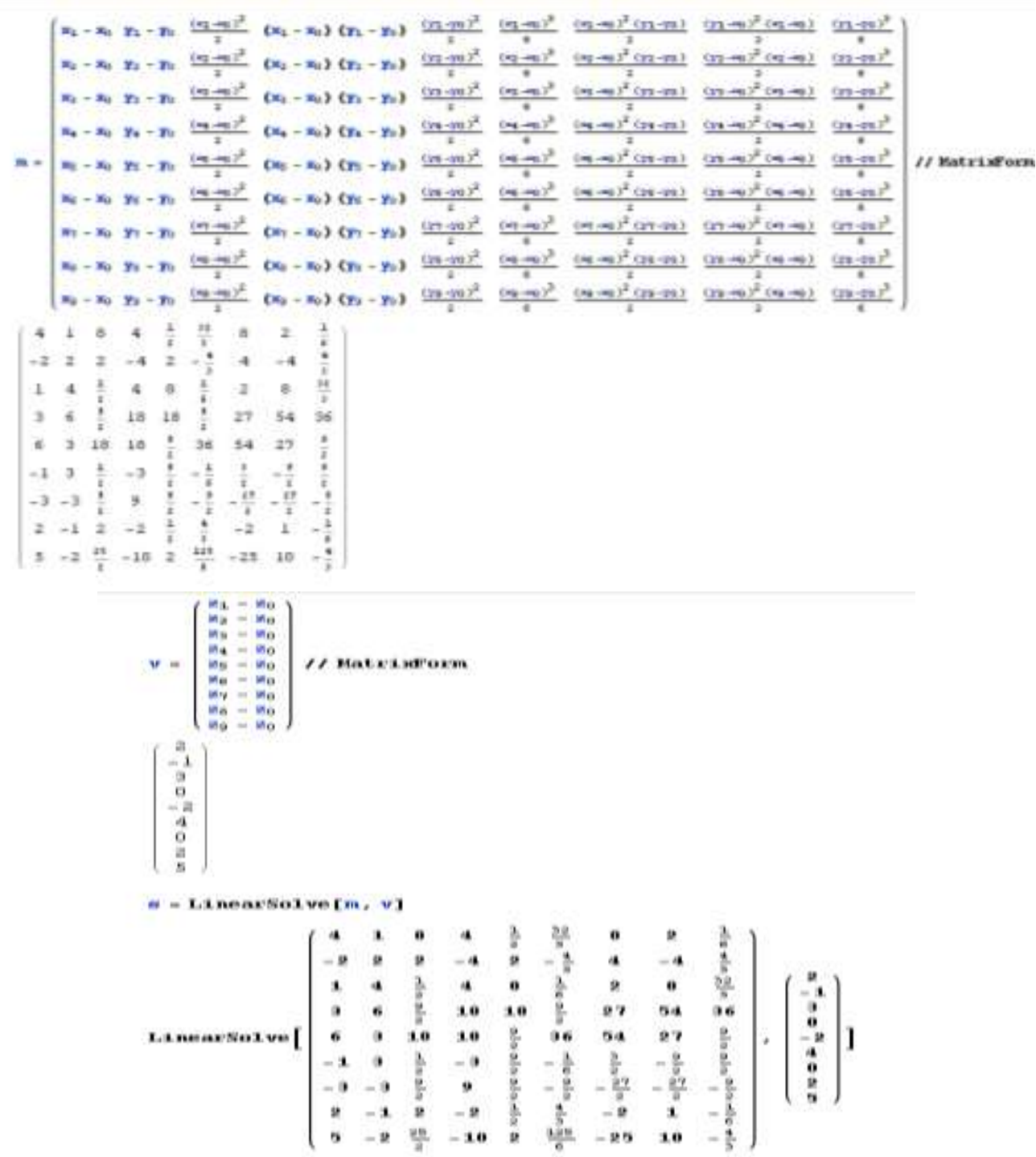


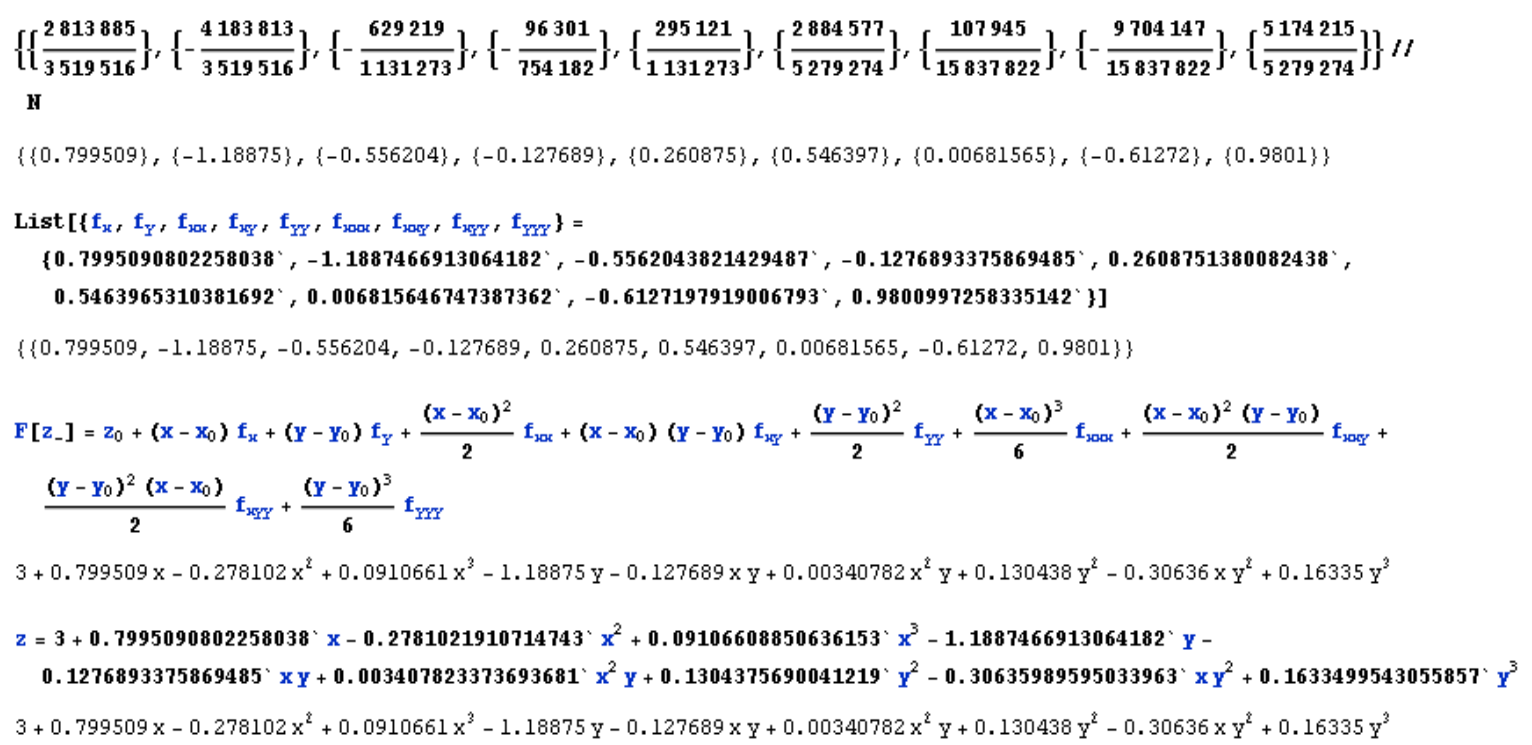

The equation of cubic patch of the surface is:

$$
\begin{aligned}
& z=3+0.7995090802258038^{\prime} x-0.2781021910714743 x^{2}+0.09106608850636153 x^{3}- \\
& 1.1887466913064182^{\prime} y-.1276893375869485 x y+0.003407823373693681 x^{2} y+0.1304375690041219^{\prime} y^{2}-
\end{aligned}
$$$$
0.30635989595033963^{`} x y^{2}+0.1633499543055857^{`} y^{3} \text {. }
$$

Or numerically:

$$
\begin{aligned}
& \mathrm{z}=3+0.799509 \mathrm{x}-0.278102 \mathrm{x}^{2}+0.0910661 \mathrm{x}^{3}-1.18875 \mathrm{y}- \\
& \quad-0.127689 \mathrm{xy}+0.00340782 \mathrm{x}^{2} \mathrm{y}+0.130438 \mathrm{y}^{2}-0.30636 \mathrm{x} \mathrm{y}^{2}+ \\
& +0.16335 \mathrm{y}^{3} .
\end{aligned}
$$

The program listed above help us to compute cubic patch of the surface for any ten points.

From this paper:

\section{Conclusions}

1- To show how to use Mathematica programs for computations the equations of the patch of the surfaces.

2- New method for designing quadratic patch of the surfaces from any six points.

3- New method for designing cubic patch of the surfaces from any ten points.

\section{References}

[1]. D. Hilbert \& S. Cohn-Vossen, The Second-Order Surfaces, $\$ 3$ in Geometry and the Imagination ( New York: Chelsea, 1999) pp. 12-19.

[2]. B. Hunt, The 27 lines on a cubic surface and Cubic surfaces, Ch. 4 and appendix B. 4 in the geometry of some special arithmetic quotients ( New York; Springer verlag, 1996) $108-67$ and $302-10$.

[3]. $\quad$ G. Alfred, Modern Differential Geometry of Curves and Surfaces (CRC Press, 1993).

[4]. L. Suresh and W. Joe, Bézier representation for quadric surface patches, Computer-Aided Design, Vol. 22, 1990, 574-579.

[5]. W. Boehm and D. Hansford, Parametric representation of quadratic surfaces, Mathematical Modeling and Numerical Analysis, Vol. 26, 1992, 191-200

[6]. W. Boehm and D. Hansford, in G. Farin editor, Bezier patches on quadratics, NURBS for Curve and Surface Design (SIAM, Philadelphia, 1991) $1-14$

[7]. I. D. Faux and M. J. Pratt, Computational geometry for design and manufacture, Mathematics and its Applications (Ellis Horwood limited, New York-Chichester, 1979).

[8] R. B. M. Amer and M. A. Abd El- Mageed, Using Stirling's Interpolation to Find Gauss and Mean Curvature for the Surface, IOSR Journal of Mathematics, 4 (2), 2012, 52-61. 